\title{
Expert review on coronary calcium
}

\author{
Matthew J Budoff \\ Khawar M Gul \\ Division of Cardiology, Saint John's \\ Cardiovascular Research Center, Los \\ Angeles Biomedical Research Institute \\ at Harbor-UCLA, Torrance, California, \\ USA
}

\begin{abstract}
While there is no doubt that high risk patients (those with $>20 \%$ ten year risk of future cardiovascular event) need more aggressive preventive therapy, a majority of cardiovascular events occur in individuals at intermediate risk (10\%-20\% ten year risk). Accurate risk assessment may be helpful in decreasing cardiovascular events through more appropriate targeting of preventive measures. It has been suggested that traditional risk assessment may be refined with the selective use of coronary artery calcium (CAC) or other methods of subclinical atherosclerosis measurement. Coronary calcification is a marker of atherosclerosis that can be quantified with the use of cardiac CT and it is proportional to the extent and severity of atherosclerotic disease. The published studies demonstrate a high sensitivity of CAC for the presence of coronary artery disease but a lower specificity for obstructive CAD depending on the magnitude of the CAC. Several large clinical trials found clear, incremental predictive value of CAC over the Framingham risk score when used in asymptomatic patients. Based on multiple observational studies, patients with increased plaque burdens (increased CAC) are approximately ten times more likely to suffer a cardiac event over the next 3-5 years. Coronary calcium scores have outperformed conventional risk factors, highly sensitive C-reactive protein (CRP) and carotid intima media thickness (IMT) as a predictor of cardiovascular events. The relevant prognostic information obtained may be useful to initiate or intensify appropriate treatment strategies to slow the progression of atherosclerotic vascular disease. Current data suggests intermediate risk patients may benefit most from further risk stratification with cardiac CT, as CAC testing is effective at identifying increased risk and in motivating effective behavioral changes. This article reviews information pertaining to the clinical use of CAC for assessing coronary atherosclerosis as a useful predictor of coronary artery disease (CAD) in certain population of patients.
\end{abstract}

Keywords: computed tomography, electron beam, prognosis, review, coronary artery calcification, calcium score, atherosclerosis, multi-detector computed tomography

\section{Introduction}

Screening studies to detect occult cancers, such as breast and colon cancer, are recommended in appropriate risk adults to help improve survival in these life-threatening conditions (Smith et al 2005). Although atherosclerotic vascular disease accounts for more death and disability than all types of cancer, a screening tool to detect subclinical atherosclerosis (such as coronary artery calcium) and target prevention of future cardiovascular events is only now starting to be adopted. New guidelines are calling for use of a screening test to identify high risk cohorts. The Screening for Heart Attack Prevention and Education -SHAPE Guidelines are the most recent national guidelines calling for use of atherosclerosis imaging to assist physicians in risk stratification (Figure 1) (Naghavi et al 2006).

In about $50 \%$ of individuals, the initial presentation of CAD is a myocardial infarction (MI) or cardiac death (Kannel and Schatzkin 1985). Conventional office-based risk screening methods, such as Framingham risk score (FRS) in the United States or from the Prospective Cardiovascular Münster (PROCAM) study in Germany, are among the most common and widely available for estimating multi-factorial absolute 


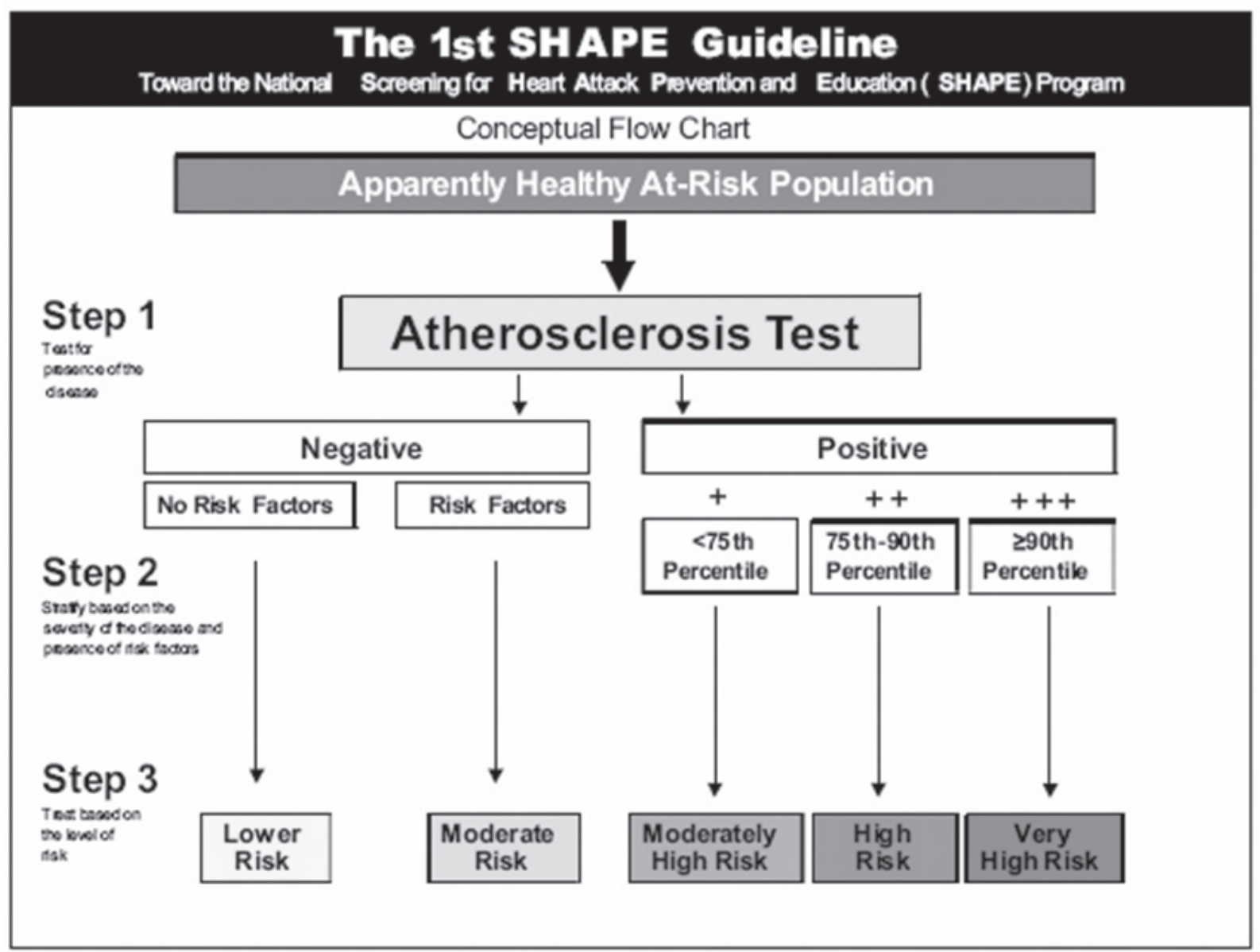

Figure I The Algorithm recommended by SHAPE (Screening for Heart Attack and Prevention). Patients with higher calcium scores get increasing therapies, as well as more diagnostic workup.

risk in clinical practice; however, these prediction models have limitations (Kannel et al 2004). These risk factor assessment tools only predict $60 \%-65 \%$ of cardiovascular risk, leaving many individuals to have cardiovascular events in the absence of traditional risk factors for atherosclerosis (Raggi 2002).

\section{Risk stratification in asymptomatic patients}

A primary recommendation of the major advisory bodies is that all adults should undergo an office-based assessment as the initial step to identify those at higher risk for a coronary event. The American Heart Association (AHA), the American College of Cardiology (ACC), and the National Cholesterol Education Program (NCEP) endorse use of the risk prediction algorithm from the Framingham Heart Study, followed by the Calcium score in certain clinical situations (Expert Panel on Detection, Evaluation, and Treatment of High Blood Cholesterol in Adults 2001).
The Framingham models create three categories of 10year risk for a future cardiovascular event: $<10 \%$ (low), $10 \%-20 \%$ (intermediate), and $>20 \%$ (high). Individuals with risk $<10 \%$ are thought to be at low risk of coronary events and current guidelines recommend reassurance without a need for further risk stratification. A sizable group of men greater than 45 years of age and women greater than 55 years of age are categorized as intermediate risk based on cardiovascular risk factors. Patients in this group currently do not qualify for the most intensive risk factor interventions, yet they may have risk factors that exceed desirable levels. It is this group that may benefit the most from further risk stratification, as CAC testing is effective at identifying increased risk and in motivating effective behavioral changes.

The Framingham score employs data from populationbased studies and does not take into account an individual's actual burden of atherosclerotic disease. Akosah and colleagues highlighted the shortcomings of Framingham assessment in a study of previously asymptomatic younger adults (men $<55$ and women $<65$ years of age), who were 
hospitalized for their first acute myocardial infarction (Akosah et al 2003). Of 222 such patients identified in a three year period, $75 \%$ would not have been considered for statin therapy according to the NCEP guidelines, suggesting missed opportunities for prevention of CHD. Furthermore, current population-based risk prediction algorithms appear to perform poorly in women (Michos et al 2006).

While a majority of events occur in the intermediate risk cohort, treating all these intermediate risk persons will be highly cost-inefficient. These patients, being asymptomatic and not high risk, will have poor long term compliance on therapy. In the Anglo-Scandinavian Cardiac Outcomes Trial (ASCOT), where hypertensives with three risk factors were randomized to a statin or placebo, 93 persons had to be treated for 3.3 years to prevent one cardiac event. This trial required 4 risk factors, so this doesn't even really address the 'intermediate risk population' (Sever et al 2003). Clearly, this population will benefit from further risk stratification, as many deemed to be at intermediate risk have no atherosclerosis (low risk) and a portion are truly high risk, and would strongly benefit from pharmacotherapy to reduce their cardiac risk.

The extent of coronary atherosclerosis, rather than the severity of stenosis, is the most important predictor of death due to acute MI or sudden cardiac death (Schmermund et al 1997). The quantification of atherosclerotic burden has become vital to proper risk stratification, especially in the intermediate risk population (Mieres et al 2005). Established noninvasive methods of evaluating CAD, such as stress testing, generally identify only patients with advanced atherosclerotic disease leading to a flow-limiting coronary stenosis and myocardial ischemia (Greenland and Gaziano 2003; Rumberger et al 2005). The prognosis of CAD, however, is more closely related to atherosclerosis plaque burden and stability than the extent of a particular stenosis (Stary et al 2003). There is growing interest in quantifying and characterizing atherosclerosis in its preclinical, pre-flow limiting phase so that appropriate preventive strategies can be instituted before an adverse event occurs (Patel et al 2004). Cardiac CT, now with both multi-detector row computed tomography (MDCT) and EBT, enables the acquisition of thin slices of the heart and coronary arteries gated to diastole, to minimize coronary motion. Both are sensitive noninvasive techniques that can detect and quantify coronary calcium, a marker of atherosclerosis (Nasir et al 2003; Greenland and Kizilbash 2005). If used in the appropriate population, it may assist clinicians to identify individuals who would benefit from more aggressive primary prevention strategies (Budoff et al 2006).

\section{Coronary calcium - basis and background}

The presence of calcium in coronary arteries is pathognomonic of atherosclerosis (Budoff et al 2006). The close correlation between the atherosclerotic plaque burden and the extent of CAC has been confirmed both by histopathology and intravascular ultrasound (Mintz et al 1997; Baumgart et al 1998). The total CAC score measured represents an anatomic measure of overall cardiac plaque burden (Figures 2-4) (Rumberger et al 1995; Baumgart et al 1997; Mintz et al 1997; Budoff et al 2006.

\section{Coronary artery calcification and obstructive disease}

Coronary calcified plaque by cardiac CT has a high sensitivity and negative predictive power for obstructive CAD but limited specificity. CAC can assist the clinician in effectively 'ruling out' angiographically significant CAD in symptomatic patients. In a study of 1851 patients undergoing angiography and CAC (Budoff et al 2002), a negative score (no calcification) was highly associated with no obstruction on angiography (negative predictive power of $98 \%$ ), and the CAC markedly improved the model to predict the presence of obstructive disease. The authors suggested that $\mathrm{CAC}$ can be used as a filter prior to angiography, with negative studies (zero scores) not continuing to invasive angiography. Recent guidelines state that CAC is "sufficiently accurate for predicting the presence of angiographic stenosis" and support the use of CAC in symptomatic persons (O'Rourke et al 2000; Budoff et al 2006). Since calcified plaque may be present in nonobstructive lesions, the presence of CAC in asymptomatic persons does not provide rationale for revascularization, but rather risk factor modification and possible further functional assessment. Clinicians must understand a positive calcium scan indicates atherosclerosis, but most often, no significant stenosis. The absence of coronary calcium is most often associated with a normal nuclear test and no obstructive disease on angiography.

Miranda et al (2000) demonstrated a relationship between $\mathrm{CAC}$ and nuclear testing in 233 consecutive asymptomatic patients who had CAC and SPECT. No patients with a CAC $<100$ had an abnormal SPECT, whereas 4.1 percent with a moderate (101 to 400) CAC and 15 percent with a CAC $>400$ had an abnormal SPECT. The best CAC cutoff for predicting an abnormal SPECT in this study was a score $>400$ (Miranda et al 2000). 


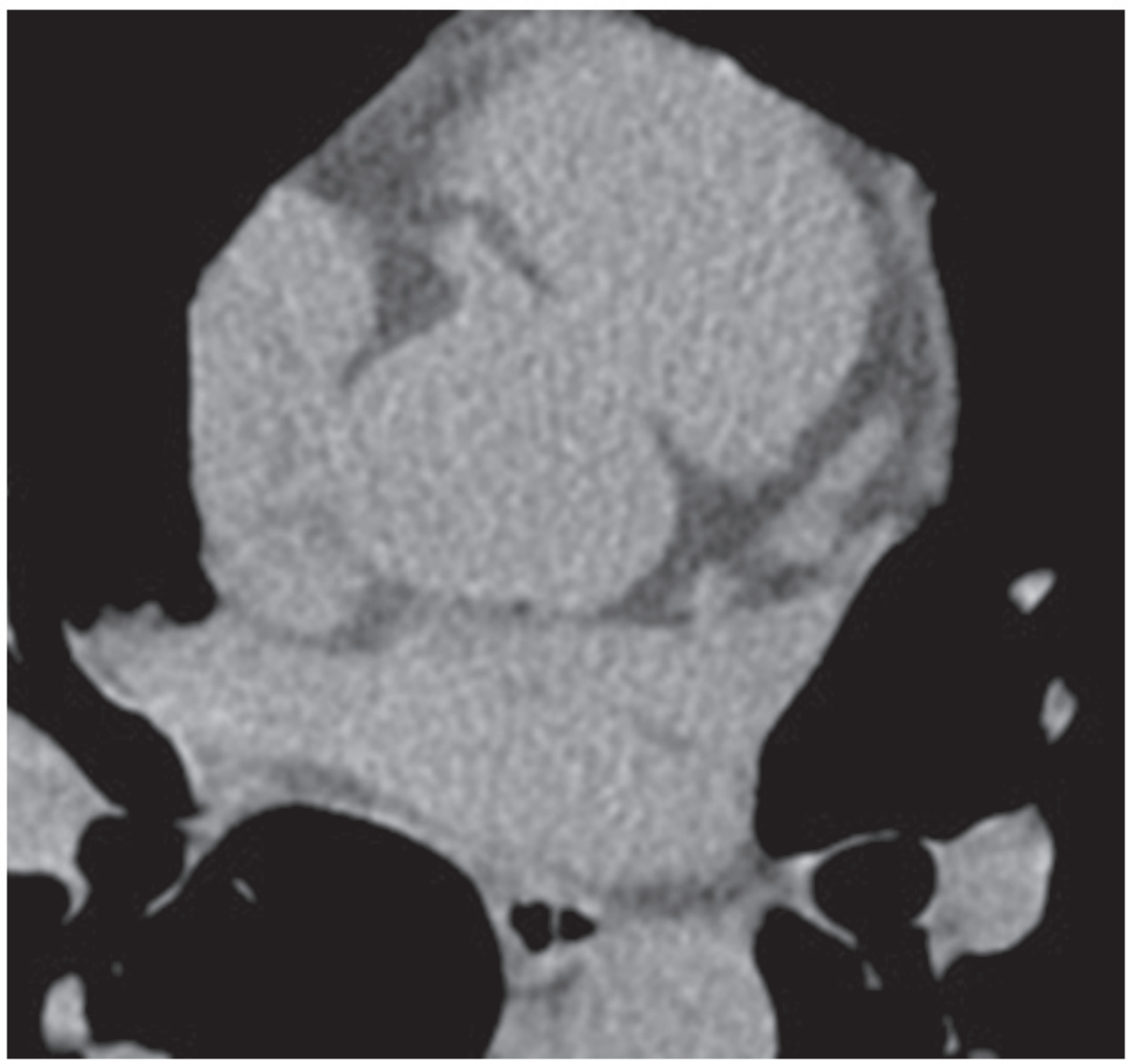

Figure 2 56-year-old male with family history of heart disease, found to have no coronary calcification. This person was subsequently not treated with statin therapy, after being assessed as low risk by CT imaging.

A recent study of 1,195 patients who underwent CAC measurement with CAC and myocardial perfusion SPECT (MPS) assessment demonstrated that CAC was often present in the absence of MPS abnormalities (normal nuclear test), and that $<2 \%$ of all patients with CAC $<100$ had positive MPS studies (Berman et al 2004). This is supported by the other published reports and is synthesized in a recent appropriateness guideline from the American Society of Nuclear Cardiology and the American College of Cardiology (Brindis et al 2005). The ACC/ASNC appropriateness criteria suggest that a low score precludes the need for MPS assessment, and a high score would warrant further assessment. These criteria suggest nuclear testing may generally be inappropriate in patients with calcium scores $<100$, as the probability of obstruction or abnormal scan is very low. A person with an Agatston score $>400$ may benefit from functional testing to detect occult ischemia. The use of functional testing is paramount to determining the need for revascularization, as functionally insignificant lesions do not benefit from revascularization, and a negative nuclear test carries by itself, a low prognostic risk.

\section{Prediction of future cardiac events with coronary calcium scores in symptomatic persons}

The ability of CAC to predict future coronary events in symptomatic persons has been demonstrated in multiple studies. A multicenter study of 491 patients undergoing coronary angiography and EBT scanning found that higher CAC were associated with an increased risk of coronary events over the next 30 months as compared to patients in the lowest quartile of score (odds ratio $10.8,95 \%$ confidence interval 1.4 to 85.6 ) (Detrano et al 1996). The atherosclerotic plaque burden, not stenosis severity, was a more important marker of disease. CAC was a stronger independent predictor of future events than a sum of all of the conventional risk factors combined (Kennedy et al 1998).

Keelan et al (2001) followed 288 symptomatic persons who underwent angiography and calcium scanning for a mean of 6.9 years. They found age and CAC were the only independent predictors of future hard coronary events (RR 3.20, 95\% CI 1.17-8.71). Only 1 in 87 patients with 


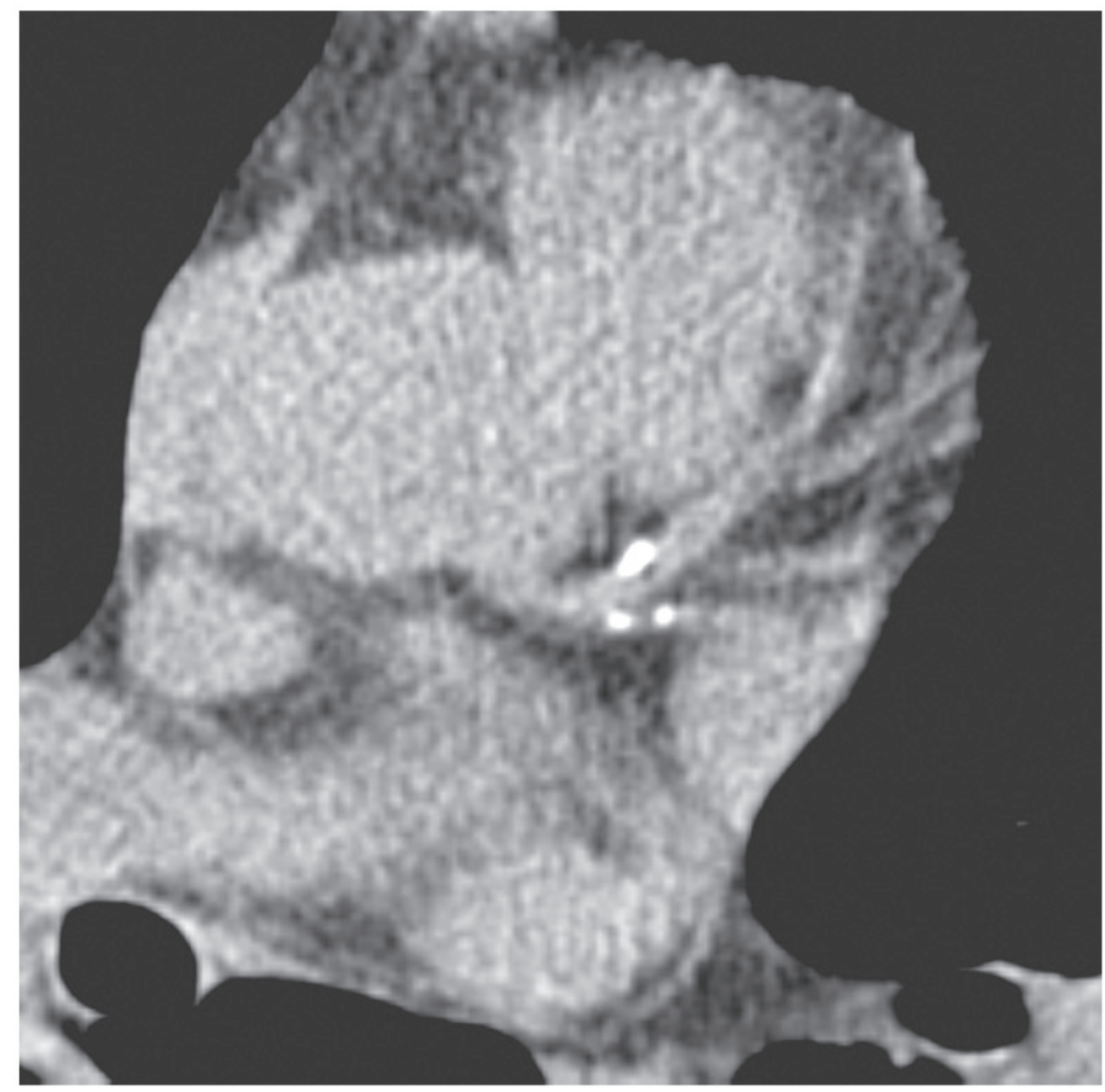

Figure 3 62-year-old sister of patient from Figure 2, found to have moderate calcifications and started on statin and aspirin therapy, in addition to lifestyle modification counseling.

CAC $<20$ experienced a subsequent hard event during follow-up. Most important of all, after stepwise multivariable analysis, neither angiographic stenosis nor conventional coronary risk factors (except age) predicted events.

A study of 192 patients observed for an average of 50 \pm 10 months showed that absolute calcium score values were strongly related to the occurrence of hard events $(\mathrm{p}<0.001)$ and all cardiovascular events ( $\mathrm{p}<0.001$ ) (Georgiou et al 2001). Patients with high scores were 13.2 times more likely to suffer a cardiac event than those with low or zero scores.

\section{Prediction of events in asymptomatic persons using coronary calcium}

The extent of CAC has been shown in several studies to predict cardiac events in asymptomatic individuals (Table 1). Raggi et al reported the occurrence of hard CHD events (MI or CHD death) in 632 individuals followed for
32 months. They found that $70 \%$ of patients with hard CHD (MI and coronary death) events had a CAC $\geq 75$ th percentile, whereas only $25 \%$ of patients without an event had CAC in this range $(p<0.0001)$ (Raggi et al 2003). For the Framingham risk score, the receiving operator characteristic (ROC) curve concordance index (C-index) for the estimation of death was $0.67(95 \% \mathrm{CI}=0.622-0.722$, $\mathrm{p}<0.0001)$ for women and $0.68(95 \% \mathrm{CI}=0.640-0.727$, $\mathrm{p}<0.0001)$ for men. When calcification was added to this analysis, a significant improvement in death estimation was observed for both women $(p<0.0001)$ and men $(\mathrm{p}<0.0001)$. The $\mathrm{C}$-index increased to $0.75(95 \%$ $\mathrm{CI}=0.702-0.799)$ with use of CAC.

In 2003, Shaw and colleagues reported the relationship of CAC to all-cause mortality in the largest cohort studied to date, consisting of 10,377 asymptomatic individuals ( $40 \%$ women), followed for an average of $5 \pm 3.5$ years. In both men and women, CAC was an independent predictor of death $(p<0.001)$, and the risk increased proportionally to the baseline calcium scores (risk factor adjusted relative risk 


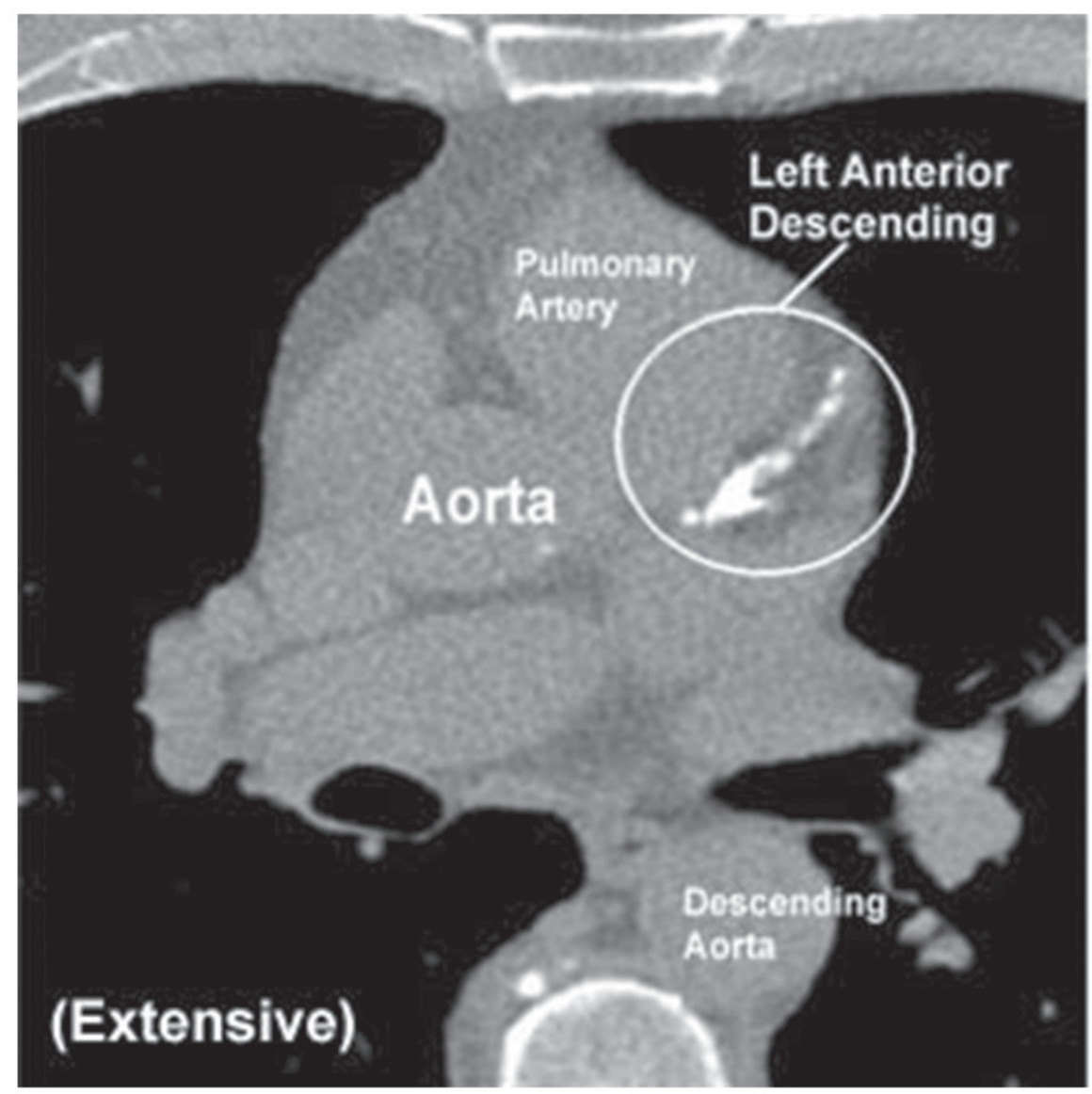

Figure 4 Extensive calcifications, warranting aggressive medical management for atherosclerosis.

of 1.6, 1.7, 2.5, and 4 for CAC 11-100, 101-400, 401-1000, and greater than 1000 respectively) (Shaw et al 2003). This large observational data series shows that coronary calcium provides independent incremental information in addition to traditional risk factors in the prediction of all-cause mortality. Further follow up in another cohort of 25,000 persons demonstrates increasing mortality rates with increasing calcium scores (Figure 5).

Wayhs et al (2002) evaluated asymptomatic individuals with a very high CAC score $(\geq 1000)$ followed for $17 \pm 11$ months. Patients with scores $>1000$ experienced an annual event rate of $25 \%$ for hard cardiac events (MI and death). Patients with events had significantly higher CAC scores than those without events despite similar age and risk factor distribution.

A study demonstrated the risk stratification in 510 uncomplicated type 2 diabetes in a prospective evaluation of measured risk factors, coronary artery calcium and myocardial perfusion scintigraphy (Anand et al 2006). Multivariable logistic regression analysis showed that CAC score was the only predictor of myocardial perfusion abnormality $(\mathrm{p}<0.001)$. In the multivariable model, the CAC score and extent of myocardial ischemia were the only independent predictors of outcome $(\mathrm{p}<0.0001)$. ROC analysis demonstrated that CAC predicted cardiovascular events with the best area under the curve (0.92), significantly better than the United Kingdom Prospective Diabetes Study Risk Score (0.74) and Framingham Score $(0.60, p<0.0001)$. The relative risk to predict a cardiovascular event for a CAC score of 101-400 was 10.1, and increased to 58.1 for scores $>1000$ $(\mathrm{p}<0.0001)$. The relative risk for ischemic burden was 5.5 for $1 \%-5 \%$ burden, increasing to 12.3 for an ischemic burden $>5 \%(p<0.0001)$. No cardiac events occurred in subjects with zero or low calcium scores $(<10)$ up until 2 years of follow-up. CAC and MPS findings were synergistic for the prediction of short-term cardiovascular events. The authors concluded that subclinical atherosclerosis, measured by CAC imaging, is superior to the established cardiovascular risk factors for predicting silent myocardial ischemia and short-term outcomes. 


\section{Cumulative Survival by Coronary Calcium Score}

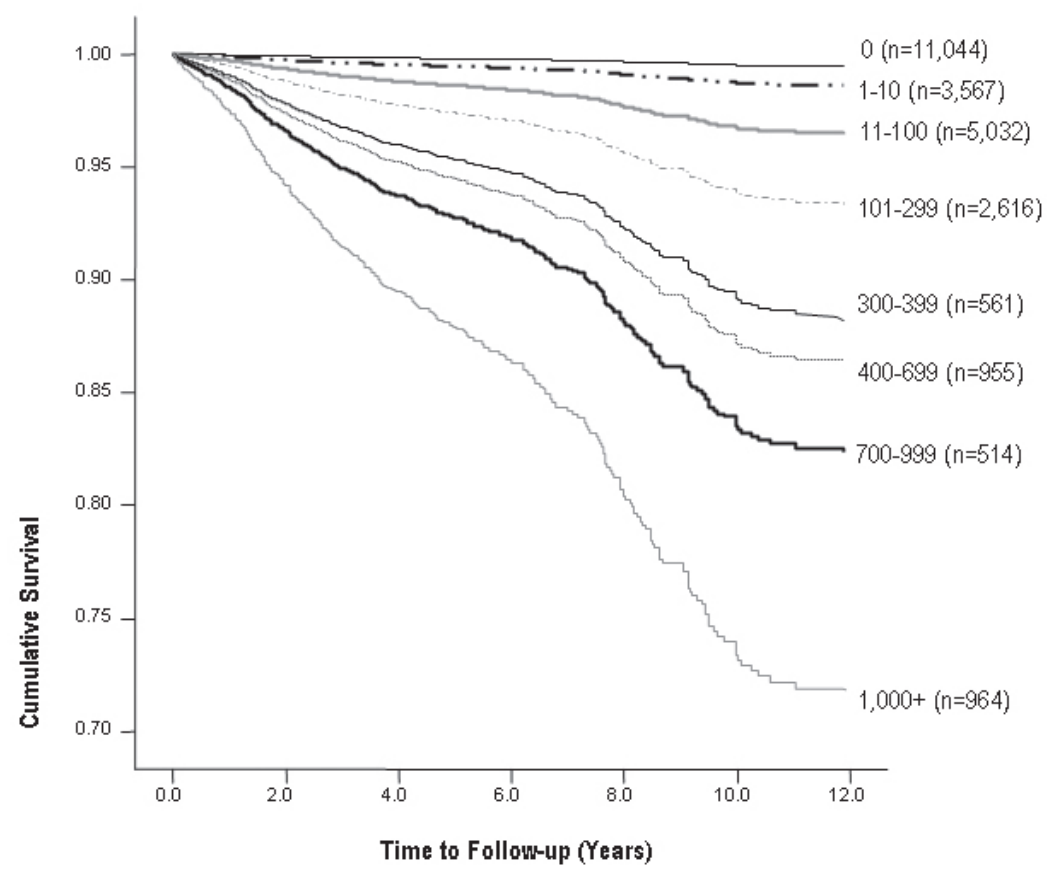

Figure 5 Ten-year follow up for all cause mortality. Patients with scores $>1000$ had a $26 \%$ mortality rate, multiples higher than patients without coronary calcification.

Arad et al (2000) in a prospective study following over 1100 patients for 3.6 years, demonstrated CAC to be the strongest predictor of future cardiac events, with patients in the highest score category over 20 times more likely to suffer a cardiac event (odds ratio: 22.3, CI 5.1-97.4).

The South Bay Heart Watch was the first study to compare the prognostic ability of $\mathrm{CAC}$ and highly-sensitive $\mathrm{C}$-reactive protein (CRP) (Park et al 2002). This study demonstrated that $\mathrm{EBT}$ derived $\mathrm{CAC}$ were incremental in predicting cardiac risk, the relative risk (RR) of hard cardiac events (MI and death) increased from 1 to 4.9 with increasing calcium tertiles ( $p=0.005$ ), while highly sensitive CRP failed to add independent prognostic value for cardiac events (myocardial infarction and death). This study has been followed out to 7 years, demonstrating consistent predictive abilities of CAC for future cardiac events (Greenland et al 2004).

The St. Francis Heart Study, a prospective observational study of almost 5000 persons, evaluated coronary calcium scores, risk factors and C-reactive protein, and included these variables in the multivariate model. (Arad, Goodman, et al 2005). A calcium score $>100$ (moderate plaque present) predicted all atherosclerotic cardiovascular disease events and the sum of non-fatal MI and coronary death events with relative risks of 9.5 to 10.7 at 4.3 years, as compared to patients with scores $<100$. This prospective study strongly demonstrated the ability to utilize this test to rule out patients who don't need therapy. Persons with zero scores had event rates of one per one thousand persons per year, demonstrating a low risk group that may be effectively treated with lifestyle changes. Similar to Park et al (2002) EBT was predictive of coronary events, while highly sensitive CRP was not.

Wong et al (2000) in a study of almost 1000 persons followed for three years, demonstrated that the calcium score predicted events independently of age, gender, and other cardiovascular risk factors (RR 8.8 for scores in the top quartile, after adjusting for conventional risk factors). The presence of $\mathrm{CAC}$ and increasing score quartiles were related to the occurrence of new MI $(\mathrm{p}<0.05)$, revascularization $(\mathrm{p}<0.001)$ and total cardiovascular events $(\mathrm{p}<0.001)$.

Kondos et al (2003) reported a 37-month follow-up on 5,635 initially asymptomatic low to intermediate risk adults (mean age $51 \pm 9$ years). Patients with scores $>170$ had a relative risk for developing hard cardiac events of 7.2 (95\% CI, 2.0-26.2), after multivariable analysis was performed with adjustment for age and other CAD risk factors. The presence of CAC provided incremental prognostic information over age and other risk factors. 
In the Rotterdam Coronary Calcification Study, 2013 participants (mean age $71 \pm 5.7$ years) were evaluated. (Vliegenthart, Oudkerk, et al 2002). Compared to subjects in the lowest calcium score category (0-100), the age-adjusted odds ratio for $\mathrm{MI}$ in the highest score category was $7.7(95 \%$ CI 4.1-14.5) for men and 6.7 (95\% CI 2.4-19.1) for women. It was also reported that subjects were three times more likely to have experienced a stroke with calcium scores $>500$ (OR $3.3,95 \%$ CI 1.5-7.2) as compared to subjects in the lowest calcium score category $(<100)$, and adjusting for carotid intimal thickness and cardiovascular risk factors did not alter the risk estimates (Vliegenthart, Hollander, et al 2002). In this first study reporting the prognostic ability of both IMT and $\mathrm{CAC}$, only CAC was predictive. Further studies with both modalities are ongoing.

\section{Limitations}

Although over 1,000 articles have appeared over the past two decades about the use of cardiac CT in coronary calcification imaging, a number of questions remain unanswered regarding its application in the clinical setting. There is now direct data on the outcome of intervention based on the calcium score, the St. Francis Heart Study (Arad, Spadaro, et al 2005). Treatment with atorvastatin $20 \mathrm{mg} / \mathrm{dl}$ reduced low-density lipoprotein cholesterol by $39 \%$ to $43 \%$ ( $p<0.0001$ ), and triglycerides by $11 \%$ to $17 \%(\mathrm{p}=0.02)$, while reducing clinical endpoints by $30 \%$ (6.9\% vs. $9.9 \%, p=0.08)$. Event rates were related to baseline calcium score and were reduced in a subgroup of participants with baseline calcium score $>400(8.7 \%$ vs. $15.0 \%$ [ $42 \%$ reduction], $p=0.046)$. The magnitude of the risk reductions were similar to studies of the same cholesterol lowering agent in studies published to date in primary prevention cohorts (Sever 2003). Assuming a 30\% improvement in life-expectancy associated with CAC screening, Taylor et al found that identifying patients with CAC is cost effective (Taylor et al 2005). The most costeffectiveness use of CAC would be use in deciding who does not need expensive statin therapy in the short term. Two of the largest studies with measured risk factors demonstrate a post-test probability of events of approximately $0.1 \%$ per year for persons without calcification present. Taylor et al (2005) prospectively followed 3,000 persons (mean age 43 years) for 3 years. CHD events occurred in only 2 of 1,263 participants without CAC (event rate $0.16 \%$; p $<0.0001$ ). Thus, a negative scan was associated with a $0.05 \%$ per year risk of events. In the St Francis Heart Study with 4,903 asymptomatic persons age 50 to 70 years, only $8 / 1504(0.5 \%)$ persons with scores of zero had a coronary event over the next 4.3 years; with an annual event rate of only $0.1 \%$. Two other prospective studies demonstrated no events in persons with scores of zero over 2-3 years of followup (Arad et al 2000; Becker et al 2005).

Ongoing studies should further clarify populations that would benefit from CAC scoring. The Multi-Ethnic Study of Atherosclerosis (MESA) will assess the long-term outcome of 6,814 adults aged 45-85 who were felt to be free of cardiovascular disease at baseline and underwent cardiac $\mathrm{CT}$, as well as other imaging and non-imaging tests (Bild et al 2005). This study will shed further light on the role of cardiac CT in primary prevention, as well the track calcium accumulation in the progression of atherosclerotic disease in diverse ethnic groups including African Americans, Hispanics, and Caucasians. In this study, CAC scoring is not blinded so resulting changes in treatment initiated by physicians may affect outcomes. The Heinz Nixdorf Recall Study (Schmermund et al 2002) is an ongoing study in 4800 men and women (47\% female) that will assess the natural history after calcium scoring, as neither the physician nor the patient will be aware of the calcium scores. The results of this study should be available in the next year.

\section{Recommendations from professional societies}

In the spring of 2005, an AHA Scientific Statement regarding the evaluation of coronary artery calcium in women stated, "Given the evolving literature... current data indicate that CAD risk stratification is possible. Specifically, low CAC scores are associated with a low adverse event risk, and high CAC scores are associated with a worse event-free survival." This guideline included a recommendation to measure atherosclerosis burden using CAC, in clinically selected intermediate-CAD risk patients (eg, those with a $10 \%$ to $20 \%$ Framingham 10 -year risk estimate) to refine clinical risk prediction and to select patients for altered targets for lipid-lowering therapies (Mieres et al 2005). The European Cardiovascular Guidelines state, "The resulting calcium score is an important parameter to detect asymptomatic individuals at high risk for future CVD events, independent of the traditional risk factors." (De Baker 2003). Both the American College of Cardiology and American Heart Association (Budoff et al 2006) have recently updated their recommendations on coronary calcium at this time.

\section{Conclusion}

The vast majority of heart attacks $(60 \%-83 \%)$ occur at the site of a non-obstructive plaque (Giroud et al 1992). Exercise 
testing or pharmacologic cardiac imaging (nuclear or echo) will only diagnose high grade coronary stenoses. They will fail to identify a vast number of asymptomatic patients at risk because an obstructive coronary plaque (stenosis in the artery of $>50 \%$ severity) is most often NOT the site of the cardiovascular event (MI or sudden cardiac death) (Little et al 1988). Framingham models miss a significant portion of patients, inappropriately stratified to intermediate or low risk. New scientific statements from the AHA (Budoff et al 2006) and SHAPE (Naghavi et al 2006) support the statement from the AHA perspective paper (Greenland et al 2001), which stated, "... with a prior probability of a coronary event in the intermediate range ( $\geq 6 \%$ in 10 years but $\leq 20 \%$ in 10 years), a calcium score of $>100$ would yield a post-test probability in virtually all such patients greater than $2 \%$ per year, that is, a level similar to that in secondary prevention, or a 'coronary risk equivalent." Therefore, all patients with CAC scores $>100$ should (Figure 4) be considered for statin therapy, aspirin and possibly ACE inhibition, given the increased cardiovascular risk associated with this level of coronary atherosclerosis, concurring with the current NCEP Adult Treatment Panel (ATP) III guidelines. This will support the conclusions of the Prevention Conference V and the AHA report that high coronary calcium scores confirm increased risk for future cardiac events: "measurement of coronary calcium is an option for advanced risk assessment in appropriately selected persons. In persons with multiple risk factors, high coronary calcium scores $(\mathrm{eg},>75$ th percentile for age and sex) denote advanced coronary atherosclerosis and provide a rationale for intensified LDL lowering therapy." By identifying high-risk patients, CAC may help select those patients who would benefit most from additional testing $(\varepsilon \gamma$, non-invasive stress imaging) and intensification of medical therapy. Furthermore, the AHA (American Heart Association) scientific statement states, "A negative test (score = $0)$ makes the presence of atherosclerotic plaque, including unstable or vulnerable plaque, highly unlikely, and is consistent with a low risk $(0.1 \%$ per year $)$ of a cardiovascular event in the next 2-5 years". CAC can be used as an effective filter before considering invasive angiography.

\section{Conclusion}

It has been shown that the presence of coronary calcium is predictive of future coronary events in both asymptomatic and symptomatic populations. This technology also provides an opportunity to assess the progression of CAD non-invasively and monitor the clinical efficacy of medical therapies by tracking the changing calcium score. As clinical decision making regarding the need for medical intervention can often be uncertain in asymptomatic individuals with one or more conventional risk factors for coronary disease, a technology such as CAC will become integral.

From the available data, intermediate risk patients benefit most from further risk stratification, as CAC testing is effective at identifying increased risk and in improving adherence to statin therapy (Kalia et al 2006). Integrating evidence regarding all available techniques is needed to determine the most practical and effective system for assessing cardiac risk to optimally target and follow the effect of preventive measures.

\section{References}

Akosah KO, Shaper A, Cogbill C, et al. 2003. Preventing myocardial infarction in the young adult in the first place: how do the National Cholesterol Education Panel III guidelines perform? J Am Coll Cardiol, 41:1475-9.

Anand DV, Lim E, Hopkins D, et al. 2006. Risk stratification in uncomplicated type 2 diabetes: prospective evaluation of the combined use of coronary artery calcium imaging and selective myocardial perfusion scintigraphy. Eur Heart J, 27:713-21.

Arad Y, Goodman KJ, Roth M, et al. 2005. Coronary calcification, coronary disease risk factors, C-reactive protein, and atherosclerotic cardiovascular disease events: the St. Francis Heart Study. J Am Coll Cardiol, 46:158-65.

Arad Y, Spadaro LA, Roth M, et al. 2005. Treatment of asymptomatic adults with elevated coronary calcium scores with atorvastatin, vitamin C, and vitamin E: The St. Francis Heart Study Randomized Clinical Trial. J Am Coll Cardiol, 46:166-72.

Arad Y, Sparado LA, Goodman K, et al. 2000. Prediction of coronary events with electron beam computed tomography. J Am Coll Cardiol, 36:1253-60.

Baumgart D, Schmermund A, Goerge G, et al. 1997. Comparison of electron beam computed tomography with intracoronary ultrasound and coronary angiography for detection of coronary atherosclerosis. $J \mathrm{Am}$ Coll Cardiol, 30:57-64.

Becker A, Knez A, Becker C, et al. 2005. Prediction of serious cardiovascular events by determining coronary artery calcification measured by multi-slice computed tomography. Dtsch Med Wochenschr, 130:2433-8.

Berman DS, Wong ND, Gransar H, et al. 2004. Relationship between stressinduced myocardial ischemia and atherosclerosis measured by coronary calcium tomography. J Am Coll Cardiol, 44:923-30.

Bild DE, Detrano R, Peterson D, et al. 2005. Ethnic differences in coronary calcification: the Multi-Ethnic Study of Atherosclerosis (MESA) Circulation, 111:1313-20.

Brindis RG, Douglas PS, Hendel RC, et al. 2005. ACCF/ASNC appropriateness criteria for single-photon emission computed tomography myocardial perfusion imaging (SPECT MPI): a report of the American College of Cardiology Foundation Quality Strategic Directions Committee Appropriateness Criteria Working Group and the American Society of Nuclear Cardiology endorsed by the American Heart Association. $J$ Am Coll Cardiol, 46:1587-605.

Budoff MJ, Achenbach S, Blumenthal RS, et al. 2006. Assessment of coronary artery disease by cardiac computed tomography. A Statement from the American Heart Association Committee on Cardiovascular Imaging and Intervention, Council on Cardiovascular Radiology and Intervention, and Committee on Cardiac Imaging, Council on Clinical Cardiology. Circulation, 114:1761-91.

Budoff MJ, Raggi P, Berman D, et al. 2002. Continuous probabilistic prediction of angiographically significant coronary artery disease using electron beam tomography. Circulation, 105:1791-6. 
De Backer G. 2003. European guidelines on cardiovascular disease prevention in clinical practice. European Heart Journal, 24:1601-10.

Detrano R, Tzung H, Wang S, et al. 1996. Prognostic value of coronary calcification and angiographic stenoses in patients undergoing coronary angiography. J Am Coll Cardiol, 27:285-90.

Expert Panel on Detection, Evaluation, and Treatment of High Blood Cholesterol in Adults. 2001. Executive Summary of the Third Report of the National Cholesterol Program (NCEP) Expert Panel on detection, evaluation and treatment of high blood cholesterol in adults (Adult Treatment Panel III). JAMA, 285:2486-97.

Georgiou D, Budoff MJ, Kaufer E, et al. 2001. Screening patients with chest pain in the emergency department using electron beam tomography: a follow-up study. J Am Coll Cardiol, 38:105-10.

Giroud D, Li JM, Urban P, et al. 1992. Relation of the site of acute myocardial infarction to the most severe coronary arterial stenosis at prior angiography. Am J Cardiol, 69:729-32.

Greenland P, Gaziano JM. 2003. Clinical practice. Selecting asymptomatic patients for coronary computed tomography or electrocardiographic exercise testing. N Engl J Med, 349:465-73.

Greenland P, Kizilbash MA. 2005. Coronary computed tomography in coronary risk assessment. J Cardiopulm Rehabil, 25:3-10.

Greenland P, LaBree L, Azen SP, et al. 2004. Coronary artery calcium score combined with Framingham score for risk prediction in asymptomatic individuals. JAMA, 291:210-15.

Greenland P, Smith Jr SC, Grundy SM. 2001. Improving coronary heart disease risk assessment in asymptomatic people: role of traditional risk factors and noninvasive cardiovascular tests. Circulation, 104:1863-7.

Kalia NK, Miller LG, Nasir K, et al. 2006. Visualizing coronary calcium is associated with improvements in adherence to statin therapy. Atherosclerosis, 185:394.

Kannel WB, D'Agostino RB, Sullivan L, et al. 2004. Concept and usefulness of cardiovascular risk profiles. Am Heart J, 14:16-26.

Kannel WB, Schatzkin A. 1985. Sudden death: lessons from subsets in population studies. J Am Coll Cardiol, 5:141B-149B.

Keelan PC, Bielak LF, Ashai K, et al. 2001. Long-term prognostic value of coronary calcification detected by electron-beam computed tomography in patients undergoing coronary angiography. Circulation, 104:412-17.

Kennedy J, Shavelle R, Wang S, et al. 1998. Coronary calcium and standard risk factors in symptomatic patients referred for coronary angiography. Am Heart J, 135:696-702.

Kondos GT, Hoff JA, et al. 2003. Coronary artery calcium and cardiac events electron-beam tomography coronary artery calcium and cardiac events: a 37-month follow-up of 5,635 initially asymptomatic low to intermediate risk adults. Circulation, 107:2571-6.

Little WC, Constantinescu M, Applegate RJ, et al. 1988. Can coronary angiography predict the site of a subsequent myocardial infarction in patients with mild-to-moderate coronary artery disease? Circulation, 78:1157-66.

Michos ED, Nasir K, Braunstein JB, et al. 2006. Framingham risk equation underestimates subclinical atherosclerosis risk in asymptomatic women. Atherosclerosis, 184:201-06.

Mieres JH, Shaw LJ, Arai A, et al. 2005. The role of non-invasive testing in the clinical evaluation of women with suspected coronary artery disease: American Heart Association consensus statement. Circulation, 111:682-96.

Mintz GS, Pichard AD, Popma JJ, et al. 1997. Determinants and correlates of target lesion calcium in coronary artery disease: a clinical, angiographic and intravascular ultrasound study. J Am Coll Cardiol, 29:268-74.

Miranda RS, Schisterman EF, Gallagher AM, et al. 2000. The extent of coronary calcium by electron beam computed tomography discriminates the likelihood of abnormal myocardial perfusion SPECT (abstr). Circulation, 102(Suppl II):II-543.
Naghavi M, Falk E, Hecht HS, et al. 2006. From vulnerable plaque to vulnerable patient-Part III: Executive summary of the Screening for Heart Attack Prevention and Education (SHAPE) task force report. Am J Cardiol, 98(2 Suppl 1):2-15.

Nasir K, Budoff MJ, Post WS, et al. Electron Beam CT vs. 2003. Helical CT scans of coronary arteries: current utility and future directions. Am Heart $J, 146: 949-77$.

O'Rourke RA, Brundage BH, Froelicher VF, et al. 2000. American college of Cardiology/American heart association expert consensus document on electron-beam computed tomography for the diagnosis and prognosis of coronary artery disease. Circulation, 102:126-40.

Park R, Detrano R, Xiang M, et al. 2002. Combined use of computed tomography coronary calcium scores and C-reactive protein levels in predicting cardiovascular events in nondiabetic individuals. Circulation, 106:2073-7.

Patel SN, Rajaram V, Pandya S, et al. 2004. Emerging, noninvasive surrogate markers of atherosclerosis. Curr Atheroscler Rep, 6:60-8.

Raggi P, Cooil B, Shaw L, et al. 2003. Progression of coronary calcium on serial electron beam tomographic scanning is greater in patients with future myocardial infarction. Am J Cardiol, 92:827-9.

Raggi P. 2002. Coronary-calcium screening to improve risk stratification in primary prevention. $J$ La State Med Soc, 154:314-18.

Rumberger JA, Simons DB, Fitzpatrick LA, et al. 1995. Coronary artery calcium areas by electron beam computed tomography and coronary atherosclerotic plaque area: a histopathologic correlative study. Circulation, 92:2157-62.

Schmermund A, Baumgart D, Goerge G, et al. 1997. Coronary artery calcium in acute coronary syndromes: a comparative study of electronbeam computed tomography, coronary angiography, and intracoronary ultrasound in survivors of acute myocardial infarction and unstable angina. Circulation, 96:1461-9.

Schmermund A, Mohlenkamp S, Stang A, et al. 2002. Assessment of clinically silent atherosclerotic disease and established and novel risk factors for predicting myocardial infarction and cardiac death in healthy middle-aged subjects: Rationale and design of the Heinz Nixdorf RECALL Study. Am Heart J, 144:212-18.

Sever PS, Dahlöf B, Poulter N, et al. 2003. ASCOT. Lancet, 361:1149-58.

Shaw LJ, Raggi P, Schisterman E, et al. 2003. Prognostic value of cardiac risk factors and coronary artery calcium screening for all-cause mortality. Radiology, 28:826-33.

Smith RA, Cokkinides V, Eyre HJ. 2005. American Cancer Society Guidelines for the early detection of cancer. CA Cancer J Clin, 55:31-44.

Stary HC, Chandler RE, Dinsmore RE, et al. 2003. A definition of advanced types of atherosclerotic lesions and a histological classification of atherosclerosis. A report from the Committee on Vascular Lesions of the Council on Arteriosclerosis. American Heart Association. Circulation, 92:1355-74.

Taylor AJ, Bindeman J, Feuerstein I, et al. 2005. Coronary calcium independently predicts incidient premature coronary heart disease over measured cardiovascular risk factors. J Am Coll Cardiol, 46:807-14.

Vliegenthart R, Hollander M, Breteler MM, et al. 2002. Stroke is associated with coronary calcification as detected by electron-beam CT: the Rotterdam Coronary Calcification Study. Stroke, 33:462-5.

Vliegenthart R, Oudkerk M, Song B, et al. 2002. Coronary calcification detected by electron-beam computed tomography and myocardial infarction: the Rotterdam Coronary Calcification Study. Eur Heart J, 23:1596-603.

Wayhs R, Zelinger A, , Raggi P. 2002. High coronary artery calcium scores pose an extremely elevated risk for hard events. $\mathrm{J} \mathrm{Am} \mathrm{Coll} \mathrm{Cardiol,}$ 39:225-30.

Wong ND, Hsu JC, Detrano RC, et al. 2000. Coronary artery calcium evaluation by electron beam computed tomography and its relation to new cardiovascular events. Am J Cardiol, 86:495-8. 